P-170

\section{Melatonin as an alternative and promising therapy for people with sickle cell anemia}

\author{
Danilo Grünig Humberto da Silva ${ }^{1}$, Nayara Alves Chaves ${ }^{2}$,
} Sayuri Miyamoto ${ }^{3}$, Eduardo Alves de Almeida ${ }^{4}$

${ }^{1}$ UNESP - Universidade Estadual Paulista, Department of Chemistry and Environmental Sciences, São Josá do Rio Preto, Brazil

${ }^{2}$ UNESP - Universidade Estadual Paulista, Department of Biology, São José do Rio Preto, Brazil

${ }^{3}$ USP - Universidade de São Paulo, Institute of Chemistry, Department of Biochemistry, São Paulo, Brazil

${ }^{4}$ FURB - Fundação Universidade Regional de Blumenau, Department of Natural Sciences, Blumenau, Brazil

This study investigated possible modulatory effects of melatonin treatment on red blood cell (RBC) metabolism in an auto incubation model. The study was carried out on six healthy donors (REF) and six sickle cell anemia patients by incubating RBCs in their own plasma at $37^{\circ} \mathrm{C}$, during different times $(0,1$, 3 and 6 hours). Furthermore, the sickle cells were incubated at the presence (tSCA) or absence (SCA) of $1 \mathrm{mM}$ melatonin. We assessed incubation period and treatment effects on markers of membrane stability, hemolysis and antioxidant capacity, as well as metabolic enzymes and subproducts. We confirmed the well-known increased osmotic resistance of sickle cells. The hemolytic rate increased over time in all three groups, except in the tSCA group at 6 hours. Neither incubation nor treatment influenced AChE activity and plasma total antioxidant capacity (TAC). On the other hand, intra-erythrocyte TAC was higher in the REF after one hour of incubation. Moreover, melatonin treatment prevented NADPH cosumption and hemin formation, and it seemed to activate $\mathrm{LDH}$. The protection effects of melatonin obtained indicate a promising use of melatonin as an antioxidant and alternative treatment for people with SCA.

E-mail address: dangrunig@gmail.com

Acknowledgements

Grant: "FAPESP-CEPID-Redoxoma (2013/07937-8) and Scholarship (2015/25983-2)"

http://dx.doi.org/10.1016/j.freeradbiomed.2018.04.317

P-171

\section{Genome-scale dissection of regulatory network dys- function in tumors with high NRF2 activity}

Daniel Levings ${ }^{1}$, Xuting Wang ${ }^{2}$, Derek Kohlhase ${ }^{1}$, Douglas Bell ${ }^{2}$, Matthew Slattery ${ }^{1}$

${ }^{1}$ University of Minnesota Medical School, Duluth, MN, USA

${ }^{2}$ National Institute of Environmental Health Sciences, Research Triangle Park, NC, USA

NRF2 is a cytoprotective transcription factor, yet NRF2 hyperactivating mutations promote oncogenesis. We used an integrative genomics approach to get an unbiased view of gene dysregulation in tumors with oncogenic NRF2 mutations and identified a set of 32 direct NRF2 targets that are consistently upregulated in these tumors. This NRF2 cancer signature includes canonical redox-related NRF2 targets (e.g., NQO1) as well as several genes not previously linked to NRF2; upregulation of this gene set is independent of the organ where the tumor developed. A key distinguishing feature of the NRF2 cancer signature genes is that they are regulated by high affinity NRF2 binding sites (antioxidant response elements) that fall within DNA regions with a ubiquitously permissive chromatin signature. This implies that the NRF2 cancer target genes are broadly responsive to oncogenic NRF2 because they lack the epigenetic restraints that restrict expression of most NRF2 targets. We used this NRF2 cancer signature to infer NRF2 activity across 9000 tumors and found that high NRF2 activity is associated with decreased survival in many cancer types. Additionally, network-based screening for drivers of this universal NRF2 cancer signature has identified several novel genetic modifiers of NRF2 activity.

E-mail address: mslatter@d.umn.edu

http://dx.doi.org/10.1016/j.freeradbiomed.2018.04.318

P-172

\section{Modulation of FKBP5 Expression by cigarette smoke}

Himadri Bhatt ${ }^{1}$, Ankit Khandelwal ${ }^{1}$, Tejal Gajaria ${ }^{2}$, Kishorchandra Gohil ${ }^{3}$, Carroll Cross ${ }^{3}$, Vihas Vasu ${ }^{1}$

${ }^{1}$ Department of Zoology and 4 Institute of Interdisciplinary Studies, The Maharaja Sayajirao University of Baroda, Gujarat, India

${ }^{2}$ Central Salt and Marine Chemicals Research Institute, Bhavnagar, Gujarat, India

${ }^{3}$ Department of Internal Medicine, Genome and Biomedical Sciences Facility University of California, Davis, CA, USA

Cigarette smoke (CS) is one of the most toxic inhaled environmental stressors leading to numerous, preventable, human pathologies. Its deleterious effects on neuro-immunomodulatory systems remain to be documented. We hypothesize that CS activates hypothalamus-pituitary-adrenal axis (HPA axis) releasing glucocorticoids by adrenal cortex. FK506 binding protein 5 (FKBP5), also known as FKBP5 1 (family of immunophilins) are transcriptionally induced by glucocorticoids and is known to regulate glucocorticoid receptor (GR) responses. Increased expression of FKBP5 was reported to confer 'glucocorticoid resistance' by binding to GR and preventing GR-translocation to nucleus. Our focus on FKBP5 was stimulated by data from a genome-wide search for mRNA correlates of (CS)-responsive lung transcriptomes. Studies comparing the lung transcriptomic profile of CS-exposed C57BL6 and AJ mice to air-breathing controls showed elevated levels of FKBP5 mRNA expression in both mouse strains. FKBP5 was also seen to regulate $\mathrm{I}_{\mathrm{k}} \mathrm{B} \alpha$ and $\mathrm{p} 65$ nuclear translocation in lung cancer cell lines, suggesting a contributory role of FKBP5 in proinflammatory stimuli of CS. The data suggests that modulation of FKBP5 expression by CS may cause dysregulated GR responses and modulate inflammatory-immune pathways in lungs.

E-mail address: hima_bhatt2031@yahoo.com

http://dx.doi.org/10.1016/j.freeradbiomed.2018.04.319

\section{P-173}

\section{MP1032 - a novel anti-inflammatory drug- amelio- rates the progression of autoimmune diseases}

Wolfgang Brysch, Sara Schumann, Petra Schulz, Maliha Shah, Katia Mangano, Paolo Fagone, David Kosel, Joerg von Wegerer, Eduard van Wijk, Ferdinando Nicoletti, Astrid Kaiser

MetrioPharm AG, Berlin, Germany 